\title{
Entrevista a Juliana González
}

\author{
Paulette Dieterlen \\ y Carlos Pereda
}

\section{ómo surge en ti la vocación filosófica o más explícitamente, con qué otro tipo de vocación entra en conflicto, cómo te deci- diste a estudiar filosofía y a ser filósofa?}

Mi vocación surgió en el bachillerato. Sin embargo ya desde niña, la idea del filósofo estaba asociada a la de mi padre, quien había muerto antes de que yo naciera; así que había detrás de mí la figura o más bien el fantasma de un padre muerto, que a decir de mi madre y de todos los seres que estaban a mi alrededor, además de escritor, había sido "un filósofo". Lo cual obviamente determinó mi vida.

Por otra parte, cuando entré a la Universidad Femenina para hacer el bachillerato tuve mi primer encuentro con todo el universo de la República española. Mis primeros maestros, fueron grandes intelectuales del exilio español, todos, de primerísima línea, que te transmitían un profundo amor tanto por la literatura como por la filosofia, principalmente. Entre ellos estaban doña Juana Ontañón, Arturo Souto, Rafael Segovia, Juan Espinasa, y muchos más. Esto, más el hecho de que mi padre también había sido novelista, generó en mí una dualidad entre la filosofia y la literatura que se prolongó por mucho tiempo. Carmen Rovira, que en estas épocas era una discípula cercana a José Gaos, fue mi maestra de filosofia en el bachillerato. Y cuando ingresé a la Facultad, entré directamenté a entrevistarme con Gaos, quien sondeó mis motivos para estudiar filosofia; me hizo importantes advertencias y me orientó para escoger mis materias y mis profesores. En esas épocas existian tutorias desde los primeros estudios, decisivas, sin duda, para orientar a los alumnos.

Pero la dualidad vocacional persistió (no propiamente conflictiva), sobre todo cuando hice teatro y muy especialmente con mi cercanía al grupo que giraba en torno de la figura del gran poeta catalán Agustí Bartra (padre 
de Roger); en este grupo estaban mis amigos más cercanos de "la espiga amotinada" (Shelley, Labastida, Oliva, Laco Zepeda, Bañuelos...) Además, en ese momento, el teatro me importaba particularmente no sólo como literatura dramática sino como actriz. Hice una obra preciosa de Bartra en la que también participaron Gironella, Bartolí y Vladi. Aunque también en esas épocas había en mí una gran inquietud por la pintura. Pintaba mucho, tomé algunas clases y quizá tenía algunas aptitudes para el dibujo, en especial, pero seguramente, no vocación.

\section{¿También para la crítica?}

Hice crítica de artes plásticas para el Suplemento Cultural de Stempre!, que en aquel entonces llevaban Benítez, Vicente Rojo, Gastón García Cantú, entre otros; estuve así trabajando en esta dificilisima tarea de la crítica de arte durante una larga temporada escribiendo cada ocho días una reseña de exposiciones de pintura, sobre todo.

¿Cuándo la vas a recoger?

Algún día, quizás. Pero creo que eran reseñas bastante malas.

\section{¿Es cuando conoces a Remedios Varo?}

Sí. Primero conocí su pintura en su primera exposición de la Galería Diana -hacia 1956, si mal no recuerdo. Luego conoci a Remedios en persona. Escribí una primera nota sobre su obra en Siempre! y a raíz de esto se consolidó nuestra amistad, que duró hasta su muerte en 1962. Incluso tuve el privilegio de que ella me diera algunas clases de dibujo, o más bien de "perspectiva", en la cual, como sabemos, Remedios era absoluta maestra. No obstante, no prosperó mi inquietud por la pintura. Más bien con Remedios aprendí que no tenía nada que hacer en ese Universo, más que gozarlo para el resto de mi vida.

En la Facultad, todavía indecisa entre la filosofía y las letras, hice una carrera maravillosa: en el primer año estudié con Gaos la Metafisica de Aristóteles, traducida directamente del griego en un seminario que duró tres años. En 1957 se tradujo Ser y tiempo, lo que me permitió estudiar a Heidegger, también con Gaos. Al mismo tiempo, llevaba un curso -decisivo para mi formación- sobre la Crítica de la razón pura con Luis Villoro. En cuanto a los presocráticos y la metafisica los estudié con Nicol. Con Gallegos Rocaful, otro de los grandes filósofos del exilio que se suele olvidar, cursé las Lecciones sobre filosofía de la historia de Hegel. Pero además de estudiar filosofia, simultáneamente a mis intensos estudios filosóficos, en estos primeros años ingresé al seminario de Literatura Dramática y Teatro con Luisa Josefina Hernández; asistí a cursos de pintura contemporánea con Ida 
Rodríguez Prampolini, de historia con O'Gorman. Por eso digo que fue una carrera maravillosa. Y poco a poco fui reconociendo que detrás de todos mis diversos intereses humanisticos y artísticos había, desde siempre, la misma fundamental vocación filosófica. Creo que en el fondo nunca hubo indecisión: que era el "llamado" de la filosofia, lo que estaba debajo de todas mis inquietudes.

La siguiente pregunta está relacionada con lo que estabas comentando, ¿cuál fue tu experiencia en la Facultad en esa época?

Creo que no se puede evitar hablar de una "época de oro". A mí me tocó vivir ese momento, en verdad luminoso, extraordinario, ya no en Mascarones sino en Ciudad Universitaria. No conoci a Joaquín Xirau, ni a Morente, ni a Imaz, por ejemplo. A Mascarones sólo fui unas cuantas veces a algunas conferencias y a un examen profesional. Aun así me produjo un impacto imborrable. Cuando entré a la Facultad ésta tenía dos años de estar en Ciudad Universitaria y aún se dejaba sentir la nostalgia que tenían los maestros de eso que fue para ellos "Mascarones". Sin embargo, me toca vivir una Facultad verdaderamente excepcional, que reflejaba la cultura de México. La formación integral que yo adquirí, no sólo se debió a la diversidad de mis inquietudes, sino a la riqueza de opciones que teníamos en todos los campos: en la Historia, las Letras, la Psicologia, la Filosofia, el Teatro. Esta formación la compartíamos todos los que formábamos parte de la Facultad, ya que no sabíamos a quién escoger en medio de tal riqueza y tal calidad del profesorado.

¿Cómo fue tu encuentro tan decisivo, como todos sabemos, con Nicol?

Mi encuentro con Nicol fue desde el primer año de carrera, en su curso sobre los presocráticos. Si algo podría rescatar en retrospectiva de lo que Nicol comunicaba como profesor, es la experiencia del filosofar como algo vivo, activo, en movimiento. Lo cual se debía ante todo a que Nicol comunicaba la experiencia del filosofar por la vià del problema filosófico y del asombro filosófico. Lo que Nicol te daba, te transmitía como catedrático, era un "estado" a la vez intelectual y emocional del acto filosófico. Estábamos en un vilo el tiempo que duraba la clase. Se decía que "dramatizaba" la filosofia; y en efecto la ponía en acción, en vida. Entramos a la filosofia por la puerta grande, que es, en efecto, la del asombro filosófico. Era un catedrático impresionante. Creo que no hubo nadie que pasara por el aula en que Nicol enseñó, que no recibiera este impacto. Gaos, en este sentido, era un profesor de otro estilo, de otra manera de entender y comunicar la filosofia, con otras virtudes didácticas. Pero también era un magnífico, excepcional maestro. 
Sin embargo, no fueron sólo los cursos lo que me atrajo de la filosofía de Nicol. En 1957, cuando cursaba el segundo año de la carrera, apareció su Metafísica de la expresión, y se me solicitó una reseña de la obra; misma que nunca hice pero cuya lectura me hizo decidir con toda claridad que mis intereses filosóficos estaban cerca del autor de esas ideas. En esa época también tenía una tesis casi terminada sobre estética; había estudiado dos años de alemán y gestionaba una beca para irme a Alemania. Sin embargo, cuando lei la Metafisica de la expresión, descarté estos proyectos y quedé, a partir de entonces, intensamente afectada por los problemas de la metafísica, en su historia y en el presente. Entré asi al seminario de Nicol donde permanecí veinte años; primero como alumna de la carrera y después ya como maestra de la Facultad. El seminario de Metafisica tenía una doble virtud: por un lado, uno asistía a la gestación del pensamiento de las ideas del propio Nicol, y todos colaborábamos y participábamos de algún modo en el proceso creador. Pero, por otro lado, y al mismo tiempo, teníamos que aprender a pensar por cuenta propia y, en especial, formarnos en los clásicos. Ahí trabajamos intensamente, no sólo en los textos de los griegos sino, por ejemplo en los filósofos modernos, como Descartes, Spinoza, Leibniz, y muy señaladamente en la obra de Heidegger. En este sentido, el seminario fue enormemente formativo. Es cierto que no era privativa de Nicol la conciencia de que uno sólo se forma filosóficamente estudiando a los clásicos: éste era, desde luego, un desideratum de todos estos grandes maestros. Y lo mismo con Nicol. Los años que pasé en el seminario fueron años en que leí, de principio a fin, a muchos de los principales autores de la historia de la filosofía. Recibimos en aquel entonces una formación que bien quisiéramos tener ahora, sinceramente.

\section{¿Que pasó con tus intereses estéticos?}

Ese proyecto se quedó ahi, varado y más bien crecieron en mí, día a día, las preocupaciones existenciales. Mis grandes amigos eran también en ese entonces por un lado, José Luis Cuevas y por el otro Salvador Elizondo. Ellos representaban los mundos de vanguardia, por decirlo de alguna manera. Mis libros de cabecera en esos tiempos eran Los endemoniados de Dostoyevski y el Ulises de Joyce. Todo ello contribuyó a formar en mí una concepción del mundo que dificilmente cabe definir.

Y crecieron también mis preocupaciones éticas. Me dediqué así a la tesis de maestría, entrando directamente al análisis crítico de la filosofia existencial. "Habité" por dos o tres años la "cámara oscura" del existencialismo, en especial el de la filosofia y la literatura de Sartre, que me produjo un impacto terrible sobre todo por sus desenlaces aporéticos, precisamente para la ética.

Y para el doctorado decidi estudiar la metafisica de Nicol, tarea que me 
llevó un buen tiempo porque me remitió además a muchas lecturas; finalmente hice la tesis que luego publiqué como libro.

Después regresé a mis propias búsquedas en la ética. La ética fue el punto de confluencia de mis inquietudes artísticas y vitales con las filosóficas, de orden teórico. Y la ética que me ha importado siempre es una ética ontológica, de raíz fenomenológica y existencial; nunca me ha interesado una ética formal. En mi seminario de Ética, ya como profesora, entré de lleno a estudiar la crisis contemporánea de la ética. Este fue el tema central y este ha sido el campo de investigación en el que me he concentrado desde entonces. Investigué el problema de la moralidad de Marx, y después entramos con Nietzsche y con Freud. Los tres pensadores llamados de "la sospecha". Con Freud quedé devorada; casi siete años me llevó la lectura completa de su obra, hecha desde un punto de vista ético y ontológico. Mi primer contacto con el psicoanálisis lo tuve también en mis años de estudiante en la Facultad, muy joven, justamente a través de Santiago Ramírez padre, quien impartía, con Ricardo Guerra, un curso conjunto de "Filosofía y psicoanálisis", al cual asistí con enorme interés; por esos tiempos conocí asimismo a Erich Fromm y a Dávila, su principal discípulo, que daba clases en la Facultad. De modo que también tuve, desde mis primeros años de formación, una llamada hacia la psicología y el psicoanálisis. Y cuando entré en la obra de Freud se me abrieron otras perspectivas, nuevas "ventanas" para los problemas del hombre y de la ética. En este momento me concentré en mis propias búsquedas en la Filosofia Moral, y pude recibir otro tipo de enseñanza (menos "sublime" - diría Pereda-) y sin perder de vista mi interés principal por una fundamentación ontológica de la ética.

Creo que, en todo este tiempo, no he pensado nada que no vaya precisamente por ese camino; y si busco las bases ontológicas y antropológicas de la ética he tratado de hacerlo, muy cercanamente a los críticos de la ética; en especial, Freud y Nietzsche. Mis trabajos sobre éste último han dado lugar a varios artículos, tres de los cuales reuní en mi libro El héroe en el alma.

Por otro lado, he de decir que jamás he dejado de interesarme por Platón.

En una época, la separación entre pensamiento marxista a ultranza y el pensamiento analitico -que comenzaba a ser influyente entre nosotros- era muy fuerte. ¿Cómo te sentías investigando el problema moral en Marx, cuando desde ciertos puntos de vista éste no se planteaba, ya que las discusiones giraban más bien en torno al carácter científico del pensamiento marxista? ¿Cómo te sentías sosteniendo una posición que en realidad no se adecuaba a las dos tendencias más fuertes?

Creo que en esta panorámica general que les estoy dando, tan a grandes y burdos trazos, se puede vislumbrar un estado de soledad filosófica. Y no 
sólo eso, sino también la presencia de una especie de "estigma", por el sólo hecho de haber optado por Nicol. Primeramente, es insoslayable el hecho de que Gaos fue, y ha seguido siendo, una figura dominante dentro del mundo de la filosofía española que llega a México en el exilio. La presencia de Gaos era tan fuerte, y su influencia en sus discípulos tan decisiva, que opacó sin duda a la de Eduardo Nicol -esto sin tomar en cuenta las graves discrepancias que hubo entre ambos, sobre todo en torno a Ortega y Gasset. Y después, ya en otras épocas, lo dominante no fue la influencia del gaosismo, sino las confrontaciones entre ciertas corrientes de pensamiento. Al principio de mis estudios, quedaban los restos de la escolástica, representada por Oswaldo Robles, en choque irreconciliable con el neokantismo. (Fue por éste último que tuve una liga con la estética kantiana y un aprendizaje de la obra de Cassirer, fundamental en mi propia formación.)

Después, los neokantianos y los neotomistas dejaron de tener presencia importante en la Facultad; se disolvieron y surgió esa otra gran polaridad entre "filosofia analítica" y "marxismo". De modo que quienes nos acercába. mos a otro tipo de filosofar, que no era marxismo ni analítica, que para colmo era la "metafísica", estábamos condenados a la descalificación y al ostracismo. Cuantos estuvimos cerca de Nicol y de eso tan desacreditado y "obsoleto" como la metafísica, hemos atravesado por caminos muy dificiles y hemos tenido que hacer un gran esfuerzo sobre todo para mantener una lealtad a las propias creencias y valores filosóficos. Por fortuna, hasta la fecha la filosofia de Nicol sigue teniendo sentido para mí. En realidad ya no sé qué tanto de ella he incorporado a mis propias búsquedas -e incluso a mi vida. Llega un momento, en general, en el que ya ni siquiera citas o mencionas a aquellos de quienes te nutriste, porque son tan fundamentales para ti que forman parte de tu propio ser.

Regresando a lo que me preguntan. Si, fue muy difícil estudiar el problema de la moralidad en Marx, no sólo porque el marxismo no tiene propiamente una preocupación por esta cuestión, sino porque había que enfrentar las tendencias de un marxismo dogmático. Pues sin duda hubo épocas dog. máticas del marxismo dentro de la propia Facultad, como las hubo en el mundo, y esto no lo podemos soslayar.

Vayamos a una pregunta que nos hace bajar del cielo a la tierra jen qué sentido tu experiencia administrativa, en particular tu experiencia dificil, y al mismo tiempo gozosa, como directora de la Facultad de Filosofia y Letras, ha influido en tu vida filosófica?

Esto de lo difícil y lo "gozoso", lo dicen porque lo hemos compartido, tanto tú Carlos, como tú Paulette, que han estado a cargo del posgrado de la 
Facultad y yo en la dirección; estamos hablando, sin duda, de una experiencia compartida.

Con relación a la pregunta concreta: siento que todavía no ha llegado el tiempo en el que pueda cobrar un poco de perspectiva y hacer balance de la experiencia. Una vez que ésta termine tendría que pensar de qué manera ella ha podido afectarme en mi vida filosófica. No lo sé. Concuerdo con lo que dicen al describirla como experiencia de goce y al mismo tiempo de sufrimiento, pero no creo que pueda decir que me ha nutrido filosóficamente más que cualquier otra experiencia vital. Más bien creo que ha sido al revés. Desde que empecé con la dirección he intentado ver cómo vincular las ideas y los valores de mi mundo filosófico, con la realidad práctica y concreta del trabajo directivo, con mis responsabilidades en la Facultad, con mi participación en el Consejo Universitario, en mi diálogo con mis colegas directores, etcétera. O sea, cómo puede la filosofía llegar, de alguna forma, por remota que sea, a un universo práctico, concreto y tan complejo como es el de nuestra Universidad. Es ahí donde he sentido que esta experiencia presenta un reto muy dificil. Porque la filosofía tiene además un lenguaje abstracto, difícilmente comunicable en mundos tan diversos, en ámbitos tan diferentes como son todos aquellos que conviven en la unam. Pero siempre he sabido también que de lo que se trata, en el fondo, es de atender a problemas prácticos de orden educativo, humano en general, y de hacer expresa una idea de la Universidad y de los valores que a nuestro juicio la constituyen. Desde que entré a la dirección sentí, al principio un poco a ciegas, que mi trabajo directivo era otra modalidad de praxis dentro de mi ejercicio filosófico, análogo a la docencia, que es sin duda la principal modalidad de la praxis filosófica. A siete años de distancia veo que siempre he tenido seguridad de que hay un vínculo entre lo académico y lo académico-administrativo que corresponde de algún modo a la relación entre lo teórico y lo práctico; esta convicción ha estado siempre presente, y ha dirigido en gran medida mi quehacer cotidiano como directora.

Háblanos un poco del papel que has jugado en la reivindicación de la presencia de las humanidades en la Universidad y también de la presencia de la Facultad en el mundo cultural mexicano.

He podido constatar en estos años que la Facultad de Filosofía y Letras tiene un lugar, un sitio bien definido y respetado, $y$ juega un papel realmente importante en el contexto general de la vida de nuestra Universidad. De ahí la responsabilidad que he sentido en todas las "comisiones" de que he formado parte, en los "Colegios de directores", en el Consejo Universitario donde he tenido que llevar esa voz que se emite desde la perspectiva humanística -y también filosófica ¿por qué no? Y es absolutamente indispensable que 
esta perspectiva esté siempre presente y cuente en el rumbo de la vida universitaria.

Por otro lado, esta Facultad siempre, no sólo ahora ha tenido una resonancia en la cultura nacional, y ha sido un espacio de expresión libre y de crítica. Tiene una personalidad propia en el mundo cultural del país y la ha tenido siempre. En la actualidad no pasa otra cosa sino que, simplemente, la Facultad sigue su curso natural. No creo que nuestra gestión haya propiciado otra cosa que no corresponda al propio elán vital de esta Facultad. Consciente e intencionalmente, hemos procurado que ella sea lo que es y ha sido a lo largo de su historia. Y no podemos olvidar que la Facultad de Filosofia y Letras está intrínsecamente unida a los institutos de humanidades; no creo que se la pueda concebir sin ellos. Los profesores y los investigadores de las disciplinas humanísticas configuramos en realidad un todo unitario; y así concebida, como "Filosofia y Letras", esta Facultad sigue siendo alfa y omega de las humanidades, aun con todas sus insuficiencias y todas las nostalgias de otros tiempos que sin duda fueron más ricos y más creativos que éstos.

Para terminar, ¿cuál es, más que tu visión, tu esperanza en torno a la filosofía en México y en lengua castellana?

La esperanza, que comparto con muchos, es que de verdad lleguemos a constituir algún día una comunidad filosófica iberoamericana. Este es un empeño que ha surgido de diversos lugares y que ojalá, se logre consolidar. Algo se ha hecho al respecto. La Asociación Filosófica, por ejemplo, ha servido para unirnos con los filósofos de América Latina y de Norteamérica, y, desde luego, con los filósofos españoles. Los encuentros institucionales que hemos realizado aquí, en España, en Argentina, en Perú, en Colombia, nos cohesionan sin duda, nos comunican. Pero nos falta una enormidad para que de verdad logremos una auténtica comunidad filosófica. No nos conocemos, no hemos desarrollado una comunidad crítica. Cuando hay crítica, ésta suele ser una erística bárbara, caníbal, en donde se pone la "inteligencia" simplemente al servicio de la eliminación del otro. Lo cual, además de contrario a la genuina filosofia, me parece absolutamente estéril y simplemente devastador. No hemos desarrollado la posibilidad de conocernos en serio, de discrepar en el entendimiento y en el respeto, y nos hemos quedado en un mero rechazo refractario o en un simple desconocimiento mutuo. Lo que ocurre en los congresos, además, y que no es sólo un fenómeno de nuestro mundo académico, es que casi siempre lo que con tanta frecuencia se ha denunciado, sin que se logre superar: el puro monólogo estéril, de las supuestas -y más bien "lineales" - "mesas redondas" incapaces de generar el verdadero diálogo filosófico. En el mejor de los casos, en los congresos se recibe cierta información, pero no se produce la verdadera comunicación crítica, filosó- 
fica. Estamos atomizados, dispersos, contando con una idea superficial unos de otros. Mi esperanza es que algún día superemos esto. De acuerdo con nuestra natural comunidad lingüistica y cultural tenemos todo para hacer una genuina comunidad filosófica, para verdaderamente pensar juntos, discrepar juntos; para conocernos y trabajar en vínculo efectivo unos con otros. Se puede ver por ejemplo que sólo nos leemos cuando presentamos o reseñamos los libros recién publicados, y sólo suele ser en estas ocasiones cuando dialogamos un poco. Después, todo parece quedar igual. Escribes un libro, algunos colegas lo comentan o tu comentas los libros de otros y hasta ahí. Pero no se genera un mundo intelectual, filosófico, de crítica fecunda, que tenga una identidad, una vida propia, continua. Crear esta identidad "hispanoamericana", o "iberoamericana" es el ideal que se persigue por muchas vías. Entre ellas, por ejemplo, uno de los intentos más significativos es la Enciclopedia Iberoamericana de Filosofía. Sin embargo, por su costo y su distribución es difícil tener acceso a esta obra en México, y me imagino que otro tanto sucede en Perú o en Chile. De modo que, si bien se hacen esfuerzos tan importantes como éste, otros obstáculos dificultan la comunicación y la verdad es que en general no hemos constituido aún una cultura filosófica común. Y eso es lo que realmente nos está faltando. Independientemente de que sigamos trabajando con filósofos de otras comunidades y otras lenguas. La filosofia es obviamente universal e "internacional". Pero eso no significa que no produzcamos filosofía desde nosotros y que no hayamos de generar nuestra propia identidad cultural y nuestra comunidad por medio de una efectiva comunicación. Creo que estamos en el camino, y en México particularmente se vienen haciendo importantes esfuerzos, y en España no se diga; pero todavia nos hace falta construir esa comunidad crítica, fundada en el reciproco conocimiento. Esa es mi esperanza. 